\title{
Gene Expression Profiling of Monozygotic Twins Affected by Psoriatic Arthritis
}

This article was published in the following Dove Press journal:

Open Access Rheumatology: Research and Reviews

\author{
Maria Maddalena Angioni (D) \\ Alberto Floris $\mathbb{D}^{\prime}$ \\ Ignazio Cangemi $\mathbb{D}^{\prime}$ \\ Mattia Congia (1D) \\ Elisabetta Chessa (D) \\ Sandro Orrù (iD) ${ }^{2}$ \\ Matteo Piga (D) \\ Alberto Cauli (D) \\ 'Rheumatology Unit, Department of \\ Medical Sciences and Public Health, \\ University of Cagliari, University Clinic \\ AOU, Cagliari, 09042, Italy; ${ }^{2}$ Medical \\ Genetics, Department of Medical \\ Sciences and Public Health, University of \\ Cagliari, Cagliari, 09042, Italy
}

Correspondence: Maria Maddalena Angioni Rheumatology Unit, Department of Medical Sciences and Public Health, University of Cagliari, University Clinic AOU Cagliari, S.S. 554, Bivio per Sestu, Monserrato, Cagliari, 09042, Italy

Tel +3907051093340

Email m.maddalena.angioni@gmail.com
Introduction: Psoriatic Arthritis (PsA) is a multifactorial disease, where the relative burden of genetic, epigenetic and environmental factors in clinical course and damage accrual is not yet definitively clarified. In clinical practice, there is a real need for useful candidate biomarkers in PsA diagnosis and disease progression, by exploring its underlying transcriptomic and epigenomic mechanisms. This work aims to profile the transcriptome in monozygotic (MZ) twins with psoriatic arthritis (PsA) highly concordant for clinical presentation, but discordant for the radiographic outcomes' severity.

Methods: We describe i) the clinical case of two MZ twins; ii) their comparative gene expression profiling (HTA 2.0 Affymetrix) and iii) signal pathways and pathophysiological processes in which differentially expressed genes are involved (in silico analysis by the IPA software, QIAGEN).

Results: One hundred sixty-three transcripts and 36 coding genes (28 up and 8 down) were differentially expressed between twins, and in the brother with the most erosive form, the transcriptomic profiling highlights the overexpression of genes known to be involved in immunomodulatory processes and on a broad spectrum of PsA manifestations.

Discussion: Twins' clinical cases are still a gold mine in medical research: twin brothers are ideal experimental models in estimating the relative importance of genetic versus nongenetic components as determinants of complex phenotypes, non-Mendelian and multifactorial diseases as PsA.

Keywords: psoriatic arthritis, gene expression profiling, diseases in twin, genetic, environment-gene interaction, joint erosions

\section{Introduction}

Psoriatic Arthritis (PsA) is a multifactorial disease, where the relative burden of genetic, epigenetic and environmental factors in clinical course and damage accrual is not yet definitively clarified. Moll and Wright were the first to demonstrate familial aggregation of PsA estimating the recurrence risk ratio in first degree relatives, ${ }^{1}$ even more epidemiological evidences demonstrate a strong genetic basis to PsA. ${ }^{2}$

Genetic factors are evident from the high prevalence $(7.6 \%)$ of PsA among firstdegree relatives of PsA probands; furthermore, an excessive paternal transmission in PsA has been described. Using the CASPAR criteria, the probandwise concordance rates are approximately $11 \%$ and $5 \%$ in $\mathrm{MZ}$ and $\mathrm{DZ}$ twins, respectively. ${ }^{3-5}$ However, the role of genes in PsA development is still elusive.

The study of monozygotic twins may represent a very useful tool to understand the effects caused by genetic predisposition, epigenetic and the environmental 
factors in a disease, estimating the weight of these actors in determining the susceptibility and severity and to identify potential biomarkers in PsA.

\section{Methods}

Patients' data regarding disease presentation and its clinical and radiological course were collected from medical records updated at each follow-up visit. Patients provided informed consent and ethical approval was granted by the local NHS authority (PROMISES/AC/2018 Prot. PG/ 2018/16313), in accordance with the 1964 Helsinki declaration.

The transcriptomic analysis was carried out using the mRNAs from whole blood (Qiagen RNA blood column kit) of the couple of $\mathrm{MZ}$. Then, prior to the gene expression study, the RNA integrity and quality were checked out by Bioanalyzer (Agilent) and transcripts were analyzed by GeneChip Human Transcriptome Array 2.0 (HTA 2.0, Affymetrix); the full technical report is available in Supplementary Material. Transcripts differentially expressed between the two brothers were analyzed by the commercial software Partek Genomics Suite V 6.6, values are reported by a Fold Change cutoff \pm 2.0 (further technical details and full transcripts' list are available in Supplementary Data). Signal pathways and pathophysiological processes in which the abovementioned mRNAs are eventually involved, were in silico analyzed by the Ingenuity Pathway Analysis (IPA) software (QIAGEN Inc., https://www.qiagenbioinformatics. com/products/ingenuity-pathway-analysis).

\section{Results}

A pair of 47-year-old male MZ twins, A and B, affected by PsA according to 2006 CASPAR criteria, were studied. Twin A is a baker, while twin B is a surveyor, both were ex-smokers for about 30 years. None of the relevant was recorded in the past medical history while their family history revealed a paternal grandfather affected by cutaneous psoriasis.

Both patients presented the onset of their symptoms at the age of 17 years with symmetrical arthritis of ankles and knees. Furthermore, twin A experienced an episode of bilateral Achilles enthesitis and dactylitis of the 3rd finger of both hands, twin B presented arthritis of the left wrist: at that time, their general practitioner treated them with non-steroidal anti-inflammatory (NSAIDs) drugs only. Three years before being referred to our clinic, the two brothers were diagnosed with undifferentiated seronegative spondylarthritis and started treatment with Salazopyrine $2 \mathrm{gr} / \mathrm{die}$ and NSAIDs as needed.

When patients were firstly assessed in our tertiary Centre, they were 27 years old, the erythrocyte sedimentation rate (ESR) and C-reactive protein (CRP) levels were increased, rheumatoid factor and anti-citrullinated peptide antibodies (ACPA) were negative, family history was positive for psoriasis and HLA typing showed A03.11, B1827, CW05.07, DRB1 03.16, DQB1 02.05. Standard X-ray of the pelvis showed bilateral subchondral sclerosis at the sacroiliac joints, predominantly on the iliac side, with irregular articular contours in both patients. Thus, both twins $\mathrm{A}$ and $\mathrm{B}$ were diagnosed with PsA sine psoriasis and treated with Etanercept $50 \mathrm{mg} /$ week in combination with Methotrexate.

Despite an overall good control of peripheral and axial symptoms, because of the occurrence of anterior uveitis at the age of 36 (twin A) and 37 years (twin B), both twins switched to Adalimumab, both achieving a persistent status of minimal disease activity: ${ }^{6}$ tender joint count $\leq 1$, swollen joint count $\leq 1$, enthesitis count $\leq 1$, patient global visual analogue score VAS $\leq 20 \mathrm{~mm}$, patient pain VAS $\leq$ $15 \mathrm{~mm}$ and health assessment questionnaire $(\mathrm{HAQ}) \leq 0.5$ ) (for 10 years).

Hands and feet conventional radiography showed, in twin A, extensive and characteristic erosive and osteoproliferative alterations including typical pencil in cup lesions; tarsal and metatarsal joints of both feet were particularly involved (Figure 1A and b). In twin B, X-ray study showed less severe lesions, of note the erosive findings in the first PIP of the left foot (Figure 1D and E). Sacroiliitis was detectable in both patients (Figure 1C and F).

The gene expression analysis, the object of the present study, was performed at the age of 47 . Covering 285,000 full-length transcripts (as well as non-coding transcripts, exon-exon junctions and over 245,000 coding sequences), the transcriptomic study highlighted a list of 163 transcripts and 36 coding genes (28 up and 8 down) differentially expressed in twin A versus $\mathrm{B}(\mathrm{FC} \pm 2.0)$. Looking for the physiopathologic role of coding transcripts by combining the bioinformatic analysis with the literature, we found that Differentially Expressed Genes (DEGs) list includes some coding genes involved in inflammatory and immunity-related pathologies, such as CEACAM8, CEACAM1, CLEC4D, IGK. Again, other DEGs in the brother A are related to the extracellular matrix system and ossification and therefore associated with pathologies affecting the 
TWIN A
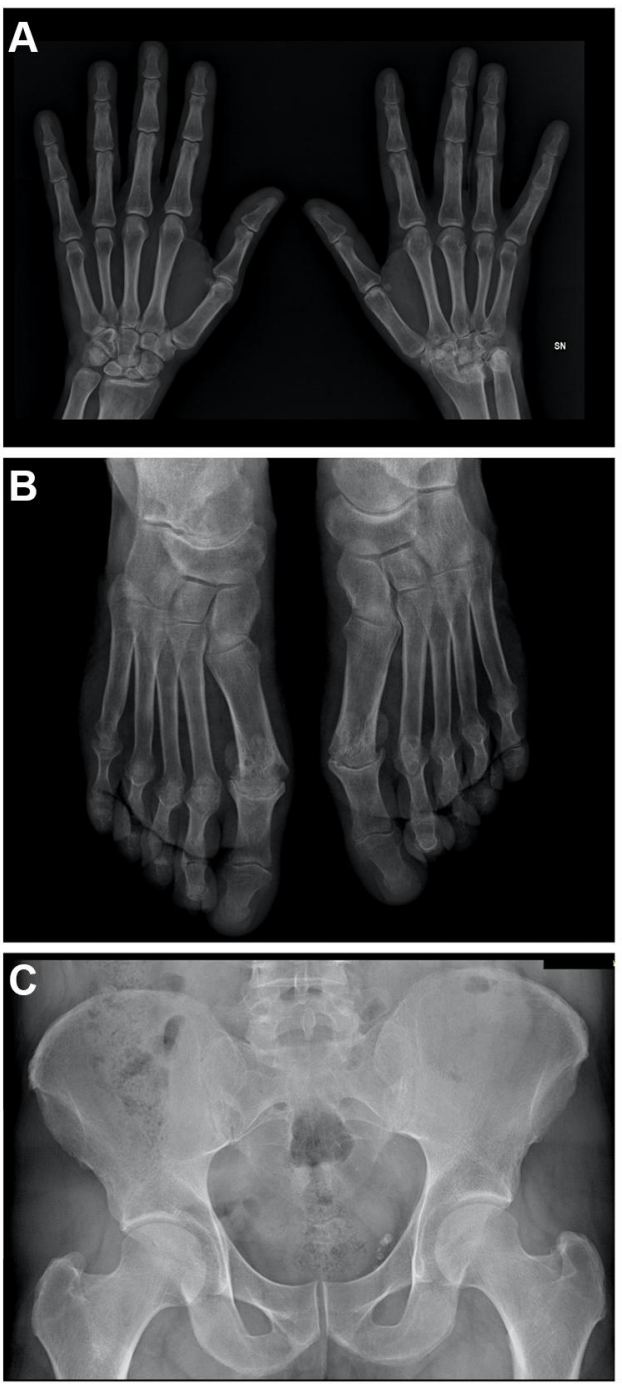

TWIN B
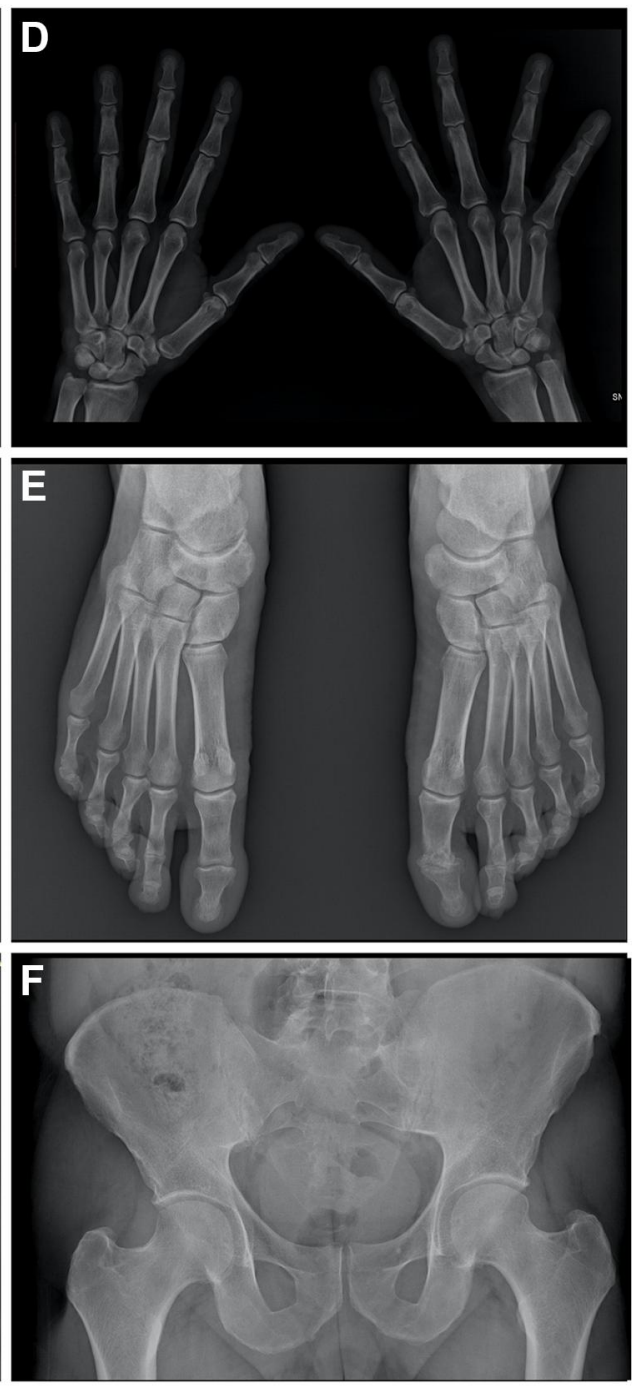

Figure I Radiographic findings in twin A and twin B. Characteristic erosive and osteoproliferative alterations including typical pencil in cup lesions are evident; tarsal and metatarsal joints of both feet were involved in twin A (A and B). In twin B (D and E) X-ray show less severe lesions, of note the erosive findings in the first PIP of the left foot (E). Sacroiliitis was detectable in both patients (C and $\mathbf{F})$.

joints, musculoskeletal and connective tissue, such as FMOD, DMD, MMP8; finally, genes that usually support cardiovascular and metabolic manifestations: ROR1, INPP5F, ACSM3, TTN and IGK (Table 1 List of selected coding DEGs between A and B twins).

Using IPA software, we examined the relationship between these DEGs to determine the most significant canonical pathways, diseases, and biological functions covered by these transcripts. The canonical pathways map representing from the top of the overrepresented network was made revealing shared biology among the identified genes (Figure 2 Overlapping Canonical Pathways map generated by IPA).
The top pathways enriched for DEGs included Myo-inositol Biosynthesis, Airway Pathology, Asthma, B Cell Receptor Signaling as shown in Figure 2. Between the top 5 diseases and biological functions, Cardiovascular, Hereditary, Skeletal and Muscular disorders, Inflammatory Response are included. Also, IPA identified significant networks associated with DEGs in twins A and B. Their associated functions were: cellular function and maintenance, cell death and survival, gastrointestinal disease, hereditary disorder, DNA replication and repair, and others represented on the picture in Supplementary Data. 
Table I List of Selected Coding Differentially Expressed Genes Between A and B Twins. Coding DEGs with a Relevant Physiopathologic Role Were Extrapolated from the Comparative Gene Expression Analysis (Fold Change Cutoff \pm 2.0; Total DEGs= 163, of Which Coding DEGs= 36), by Combining the IPA Bioinformatic Analysis with the Literature (Input: Function/ Disease Search)

\begin{tabular}{|c|c|c|c|c|}
\hline $\begin{array}{l}\text { Gene } \\
\text { Symbol }\end{array}$ & Full Name & Functions/Association to Diseases & $\begin{array}{c}\text { FC A vs B (Cutoff } \\
\pm 2.0)\end{array}$ & Refs \\
\hline ACSM3 & $\begin{array}{c}\text { acyl-CoA synthetase medium-chain family } \\
\text { member } 3\end{array}$ & $\begin{array}{l}\text { Metabolic pathways and biosynthetic processes/ } \\
\text { hypertension }\end{array}$ & 2.0 & 17,18 \\
\hline CEACAMI & Antigen-related cell adhesion molecule I & $\begin{array}{c}\text { Immunomodulatory processes/rheumatic } \\
\text { disease }\end{array}$ & 2.2 & 19 \\
\hline CEACAM8 & $\begin{array}{l}\text { Carcinoembryonic antigen-related cell } \\
\text { adhesion molecule } 8\end{array}$ & Inflammation/rheumatic disease & 2.0 & 20,21 \\
\hline CLEC4D & C-type lectin domain family 4 , member $D$ & $\begin{array}{c}\text { Inflammation/immune response/rheumatic } \\
\text { disease }\end{array}$ & 2.2 & 22 \\
\hline DMD & Dystrophin & $\begin{array}{c}\text { Cardiomyopathy/skeletal muscle tissue } \\
\text { development }\end{array}$ & 3.3 & 23 \\
\hline FMOD & Fibromodulin & $\begin{array}{l}\text { Inflammatory response/regulate TGF-beta } \\
\text { activities }\end{array}$ & 3.6 & $24-26$ \\
\hline IGKC & Immunoglobulin kappa constant & Inflammation/Amyloidosis/autoimmune disease & 2.2 & 27 \\
\hline INPP5F & Inositol polyphosphate-5-phosphatase $\mathrm{F}$ & $\begin{array}{c}\text { Carbohydrate and lipid metabolism/Cardiac } \\
\text { response to stress }\end{array}$ & 2.1 & 28,29 \\
\hline MMP8 & $\begin{array}{c}\text { Matrix metallopeptidase } 8 \text { (neutrophil } \\
\text { collagenase) }\end{array}$ & Inflammation/ossification/rheumatic disease & 2.2 & 14,15 \\
\hline RORI & $\begin{array}{l}\text { Receptor tyrosine kinase-like orphan } \\
\text { receptor I }\end{array}$ & $\begin{array}{l}\text { Inflammatory and immune response/Wnt- } \\
\text { pathway }\end{array}$ & 2.4 & $12,13,30,31$ \\
\hline TTN & Titin/Connectin & Cardiomyopathies/muscular dystrophy & 2.0 & 32 \\
\hline
\end{tabular}

\section{Discussion}

As first described by Moll and Wright in 1973, PsA can be divided into different clinical subsets and patients may demonstrate transitions between these subsets throughout the course of the disease. Furthermore, PsA has been associated with various comorbidities, including cardiovascular disease, metabolic syndrome, cancer and depression; thus, interventions that beneficially impact disease may also significantly affect morbidity and mortality. ${ }^{7}$

Monozygotic twins arise from a single cell and therefore share almost all their DNA sequence. However, the reason why the concordance rate of the autoimmune diseases in monozygotic twins is considerably below $100 \%$ is still unknown. ${ }^{8}$

With the emergence of epigenetics, researchers have begun to consider it as a critical contributing factor to discordant phenotypes. Notably, the environment plays a role in determining an individual's phenotype, and the link between environment and genetics is mediated through epigenetic mechanisms. ${ }^{9-11}$

Considering the low concordance rate in twins already reported in the literature, ${ }^{4,5}$ here we report the case of extremely clinically concordant $\mathrm{MZ}$ twins which lived in a similar environment, showing a similar clinical course but different in terms of radiographic outcomes' severity. This could be a situation in which not only the genetic components contribute to disease onset and course but also the environmental factors, eg intended as mechanical stress, perhaps played a role on the articular effort, evolving in a different severity outcome.

As reported in Table 1, the molecular profiling highlights in the twin A the overexpression of genes strictly related to inflammation and immunomodulatory processes. So, even with an almost identical genetic background and an outstanding parallel clinical course, in this clinical case, the erosive burden in PsA outcome is phenotypically 

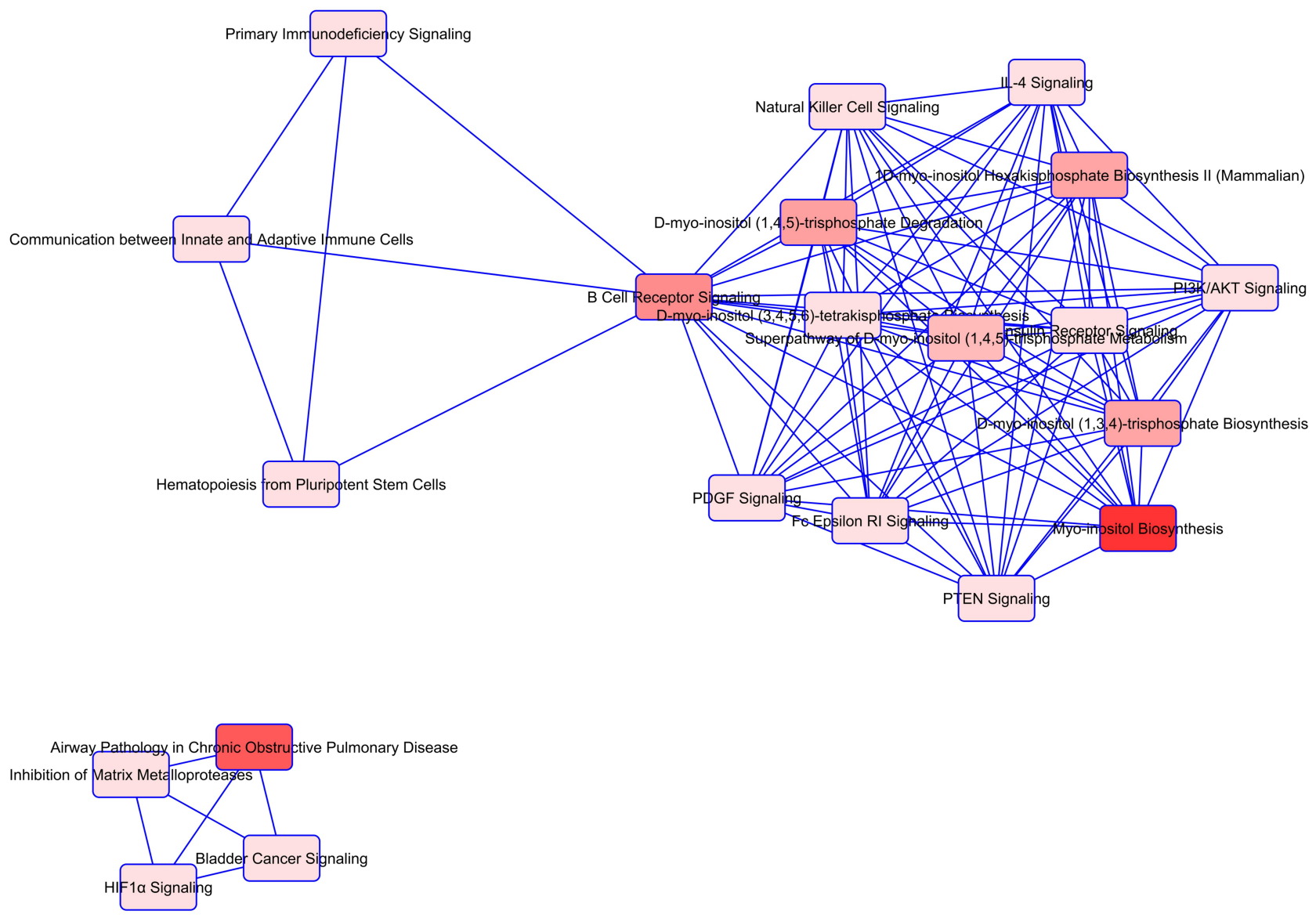

Role of NANOG in Mammalian Embry

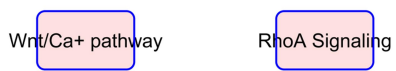

Figure 2 Overlapping Canonical Pathways map generated by Ingenuity Pathway Analysis. A pathway network was generated representing from the top of overrepresented pathways determined by IPA, to reveal shared biology among the identified candidate genes. Notes: edge-connected canonical pathways share one or more genes in common. Nodes represent pathways and bright red represents more significant canonical pathways in the gene set. The canonical pathways map was generated by QIAGEN's Ingenuity Pathway Analysis (QIAGEN Inc., https://www.qiagenbioinformatics.com/products/ingenuity-pathway-analysis).

varied between brothers, and it could depend on dysregulation of key genes as ROR1 and MMP8.

ROR1, a ROR2 closely related receptor, modulates some signaling pathways including the Hippo pathway. In particular, targets of the ROR-YAP cascade include WNT5A and connective tissue growth factor (CTGF). ${ }^{12}$ Notably, excessive activation of WNT/ $\beta$-Catenin signaling promotes a form of terminal differentiation called chondrocyte hypertrophy, ${ }^{13}$ and during skeletal development, chondrocyte hypertrophy precedes cartilage calcification and ultimately its replacement by bone.
One characteristic feature of the cartilage degeneration is the breakdown of the extracellular matrix by different matrix metalloproteinases (MMPs). The type I, II, and III collagen-breaking MMP-8 seems to play some role in Osteoarthritis ${ }^{14}$ and the expression of MMP-8 is significantly increased in the joints of mice that have collageninduced arthritis compared to healthy mice. ${ }^{15}$

Although the molecular data here described originate from a single pair of twins on chronic anti-TNF $\alpha$ treatment, which may represent a limitation and therefore needs further validations, nevertheless it is of note this clinical case of MZ twins 
surprisingly concordant for PsA onset and long-term course. In the brother (A) with the most erosive form, the molecular profiling highlights the overexpression of genes strictly related to inflammation and immunomodulatory processes but also associates with PsA comorbidities, such as cardiovascular and metabolic. ${ }^{16}$ Moreover, the presence of one disease (arthritis) within another (psoriasis, cardiovascular, metabolic, gastrointestinal) and relative severity degree may modify the molecular and clinical expression of either condition.

The present study confirms that even with an almost identical genetic background and an outstanding parallel clinical course, the erosive burden in PsA outcome may vary and could depend on a complex multi-hit model of disease, where a genetically predisposed individual encounters several environmental exposures (microbial, diet, alcohol, smoking, exercise and job) over a lifetime, which modulate the disease course.

\section{Ethics Statement}

Both patients provided informed consent for the case details and images to be published. Patients provided informed consent and ethical approval was granted by the local NHS authority (PROMISES/AC/2018 Prot. PG/2018/ 16313), in accordance with the 1964 Helsinki declaration.

\section{Acknowledgments}

We thank both the patients F.M. and F.M., who have participated in the study on a voluntary basis, and who have read and approved the manuscript.

\section{Disclosure}

Prof. Dr. Alberto Cauli reports personal fees from Abbvie, BMS, Celgene, Galapagos, Janssen, MSD, Sanofi, Alfa Sigma, UCB, and Lilly, grants and personal fees from Pfizer and Novartis, and non-financial support from Roche, outside the submitted work. The authors declare that they have no other potential conflicts of interest for this work.

\section{References}

1. Moll JM, Wright V. Familial occurrence of psoriatic arthritis.. Ann Rheum Dis. 1973;32(3):181-201. doi:10.1136/ard.32.3.181

2. Rahman P, Elder JT. Genetic epidemiology of psoriasis and psoriatic arthritis.. Ann Rheum Dis. 2005;64 Suppl 2(Suppl 2):ii37-ii41. doi:10.1136/ard.2004.030775

3. Rahman P, Gladman DD, Schentag CT, Petronis A. Excessive paternal transmission in psoriatic arthritis. Arthritis Rheum. 1999;42 (6):1228-1231.

4. Pedersen OB, Svendsen AJ, Ejstrup L, Skytthe A, Junker P. On the heritability of psoriatic arthritis. Disease concordance among monozygotic and dizygotic twins. Ann Rheum Dis. 2008;67(10):1417-1421.
5. Chandran V, Schentag CT, Brockbank JE, et al. Familial aggregation of psoriatic arthritis. Ann Rheum Dis. 2009;68(5):664-667.

6. Coates LC, Fransen J, Helliwell PS. Defining minimal disease activity in psoriatic arthritis: a proposed objective target for treatment. Ann Rheum Dis. 2010;69(1):48-53.

7. Leung YY, Ogdie A, Orbai AM, et al. Classification and Outcome Measures for Psoriatic Arthritis. Front Med. 2018;5:246.

8. Selmi C, Lu Q, Humble MC. Heritability versus the role of the environment in autoimmunity. $J$ Autoimmun. 2012;39 (4):249-252.

9. Floreani A, Leung PS, Gershwin ME. Environmental Basis of Autoimmunity. Clin Rev Allergy Immunol. 2016;50(3):287-300.

10. Xiang Z, Yang Y, Chang C, Lu Q. The epigenetic mechanism for discordance of autoimmunity in monozygotic twins. $J$ Autoimmun. 2017;83:43-50. doi:10.1016/j.jaut.2017.04.003

11. O'Rielly DD, Jani M, Rahman P, Elder JT. The Genetics of Psoriasis and Psoriatic Arthritis.. J Rheumatol Suppl. 2019;95:46-50. doi:10.3899/jrheum.190119

12. Park HW, Kim YC, Yu B, et al. Alternative Wnt signaling activates YAP/TAZ. Cell. 2015;162(4):780-794. doi:10.1016/j. cell.2015.07.013

13. Enomoto-Iwamoto M, Kitagaki J, Koyama E, et al. The Wnt antagonist Frzb-1 regulates chondrocyte maturation and long bone development during limb skeletogenesis. Dev Biol. 2002;251(1):142-156. doi:10.1006/dbio.2002.0802

14. Davidson RK, Waters JG, Kevorkian L, et al. Expression profiling of metalloproteinases and their inhibitors in synovium and cartilage. Arthritis Res Ther. 2006;8(4):R124. doi:10.1186/ar2013

15. Booth G, Newham P, Barlow R, Raines S, Zheng B, Han S. Gene expression profiles at different stages of collagen-induced arthritis. Autoimmunity. 2008;41(7):512-521. doi:10.1080/08916930802095210

16. Lubrano E, Scriffignano S, Perrotta FM. Multimorbidity and comorbidity in psoriatic arthritis - a perspective. Exp Rev Clin Immunol. 2020;3:1-10.

17. De Preter V, Rutgeerts P, Schuit F, Verbeke K. Impaired Expression of Genes Involved in the Butyrate Oxidation Pathway in Crohn's Disease Patients. Inflamm Bowel Dis. 2013;19(3):E43-E44. doi: $10.1002 /$ ibd. 22970

18. Walsh V, Somody L, Farrell A, et al. Analysis of the role of the SA gene in blood pressure regulation by gene targeting. Hypertension. 2003;41(6):1212-1218. doi:10.1161/01.HYP.0000069010.28143.5C

19. Gray-Owen S, Blumberg R. CEACAM1: contact-dependent control of immunity. Nat Rev Immunol. 2006;6(6):433-446. doi:10.1038/ nri1864

20. Zhao L, Furebring M, Xu S, Venge P. Subcellular localization and mobilization of carcinoembryonic antigen-related cell adhesion molecule 8 in human neutrophils. J Haematol. 2004;125(5):666-673. doi:10.1111/j.1365-2141.2004.04963.x

21. Seifert M, Przekopowitz M, Taudien S, et al. Functional capacities of human IgM memory B cells in early inflammatory responses and secondary germinal center reactions. Proc Natl Acad Sci U S A. 2015;112(6):E546. doi:10.1073/pnas.1416276112

22. Lorentzen JC, Flornes L, Eklöw C, et al. Association of arthritis with a gene complex encoding C-type lectin-like receptors. Arthritis Rheum. 2007;56(8):2620-2632. doi:10.1002/art.22813

23. Muntoni F, Torelli S, Ferlini A. Dystrophin and mutations: one gene, several proteins, multiple phenotypes. Lancet Neurol. 2003;2 (12):731-740. doi:10.1016/S1474-4422(03)00585-4

24. Sjöberg A, Onnerfjord P, Mörgelin M, Heinegård D, Blom AM. The extracellular matrix and inflammation: fibromodulin activates the classical pathway of complement by directly binding C1q. $J$ Biol Chem. 2005;280(37):32301-32308. doi:10.1074/jbc. M504828200

25. Zheng Z, Zhang X, Dang C, et al. Fibromodulin Is essential for fetal-type scarless cutaneous wound healing. Am J Pathol. 2016;186 (11):2824-2832. doi:10.1016/j.ajpath.2016.07.023 
26. Zheng Z, Nguyen C, Zhang X, et al. Delayed wound closure in fibromodulin-deficient mice is associated with increased TGF- $\beta 3$ Signaling. J Invest Dermatol. 2011;131(3):769-778. doi:10.1038/ jid. 2010.381

27. Streicher K, Morehouse CA, Groves CJ, et al. The plasma cell signature in autoimmune disease. Arthritis Rheumatol. 2014;66 (1):173-184. doi:10.1002/art.38194

28. Ooms LM, Horan KA, Rahman P, et al. The role of the inositol polyphosphate 5-phosphatases in cellular function and human disease. Biochem J. 2009;419(1):29-49.

29. Zhu W, Trivedi CM, Zhou D, Yuan L, Lu MM, Epstein JA. Inpp5f is a polyphosphoinositide phosphatase that regulates cardiac hypertrophic responsiveness. Circ Res. 2009;105(12):1240-1247.
30. Saito T, Fukai A, Mabuchi A, et al. Transcriptional regulation of endochondral ossification by HIF-2 during skeletal growth and osteoarthritis development. Nat Med. 2010;16:678-686.

31. Yang S, Kim J, Ryu J-H, et al. Hypoxia-inducible factor-2 is a catabolic regulator of osteoarthritic cartilage destruction. Nat Med. 2010;16:687-693.

32. Linke WA. Sense and stretchability: the role of titin and titin-associated proteins in myocardial stress-sensing and mechanical dysfunction Cardiovasc. Res. 2008;77(4):637-648.

\section{Publish your work in this journal}

Open Access Rheumatology Research and Reviews is an international, peer-reviewed, open access journal publishing original research, reports, editorials, reviews and commentaries on all aspects of clinical and experimental rheumatology in the clinic and laboratory including the following topics: Pathology, pathophysiology of rheumatological diseases; Investigation, treatment and management of rheumatological diseases; Clinical trials and novel pharmacological approaches for the treatment of rheumatological disorders. The manuscript management system is completely online and includes a very quick and fair peer-review system, which is all easy to use. Visit http://www.dovepress.com/testimonials.php to read real quotes from published authors. 